\title{
Systematic Framework for Reflectarray Synthesis Based on Phase Optimization
}

\author{
J. Álvarez, M. Arrebola, R. G. Ayestarán, and F. Las-Heras \\ Área de Teoría de la Señal y Comunicaciones, Departamento de Ingeniería Eléctrica, Universidad de Oviedo, Edificio Polivalente, \\ Modulo 8, Campus Universitario de Gijón, Asturias, 33203 Gijón, Spain
}

Correspondence should be addressed to J. Álvarez, jalvarez@tsc.uniovi.es

Received 2 March 2012; Revised 4 June 2012; Accepted 5 June 2012

Academic Editor: Raphaël Gillard

Copyright () 2012 J. Álvarez et al. This is an open access article distributed under the Creative Commons Attribution License, which permits unrestricted use, distribution, and reproduction in any medium, provided the original work is properly cited.

\begin{abstract}
A new systematic synthesis framework for reflectarray antennas is discussed. Optimization based on the Levenberg-Marquardt algorithm is used to obtain the phase distribution of the reflection coefficients required on the reflectarray surface, in order to achieve the pattern specifications. A Local Multipoint Distribution System (LMDS) base station working in the 24.5-26.5 GHz frequency band has been proposed to evaluate the method. The 3D requirements are defined by the combination of the elevation and templates and considering a maximum acceptable ripple in the beam shaping. Some illustrative results are obtained.
\end{abstract}

\section{Introduction}

Printed reflectarray antennas are very attractive alternatives to classic reflectors and phased arrays in many applications, such as beam shaping and electronic beam control [1]. They present low profile, mass, and volume and offer an easy manufacturing process. In general, a reflectarray consists of a planar array of printed elements illuminated by a primary feed, typically a horn antenna. Each element of the reflectarray should introduce a phase shift into the impinging wave from the feed, in order to obtain a beam shaped or focused in a given direction. The amplitude of the field reflected by each cell of the reflectarray is imposed by the illumination provided by the primary feed and the ohmic losses of the reflectarray.

The possibilities of contoured and focused beam reflectarrays are interesting in a wide range of applications. Multiple-beam antennas typically based on large-phased arrays are used in radar and communications applications $[2,3]$ or reflectors with feed-horn clusters and mechanical devices [4]. Moreover, reflectarrays can be a suitable technology for multibeam antennas, considering one feed per beam in order to obtain focused beams [5]. Eventually, single offset reflectarrays have been demonstrated for real DBS (Direct Broadcast Satellite) mission requirements [6, 7].
Two tasks should be carried out in the design of a contoured beam reflectarray. The phase distribution of the reflection coefficient to obtain the required beam shaping should be synthesized. Then, the dimensions of the elements should be adjusted element by element in order to match the phase shift requirements. Some phase-only synthesis methods have been proposed in order to obtain the phaseshift of the reflectarray cells, considering the large numbers of unknowns in the problem and avoiding the local minima [810]. However, these approaches do not provide a systematic synthesis framework for reflectarrays, requiring different combinations of techniques in order to adapt the method to the problem to be addressed.

In this paper, a phase-only optimization method is proposed as a systematic framework able to deal with general reflectarray synthesis problems, avoiding the need to control the process while the optimization is being carried out. In order to evaluate its performance, it has been used to obtain shaped-beam reflectarrays for a Local Multipoint Distribution Service (LMDS) base station application [11], squared cosecant in elevation and sectored in azimuth. A reflectarray has been synthesized at the central frequency of the application band $(25.5 \mathrm{GHz})$, considering the illumination of a pyramidal horn antenna as primary feed. The synthesis method is based on the definition of a proper cost function, 
where the allowed minimum and maximum levels at each angular direction of the far field are previously established $[12,13]$. The function is then minimized according to the required phase values, using an optimization method based on the Levenberg-Marquardt (LM) algorithm [14]. Some well-known optimization and random search algorithms exist in the scientific literature able to solve this problem, such as Newton-Raphson (NR) [15], Genetic Algorithms [16], or Differential Evolution Algorithm [17], but LM has been chosen as a reasonable tradeoff between complexity and performance on synthesis tasks, due to its robustness in the resolution of nonlinear least squared problems [18]. Moreover, this method has been found useful in some synthesis problems, dealing with phase-only optimization [19], or magnitude and phase synthesis of the feeding weights [20] when it is applied to linear arrays. In this paper, using and adequate cost function, the method can be applied to a planar reflectarrays, subject to demanding full $2 \mathrm{D}$ requirements, such as the specifications of an antenna for an LMDS central station.

The proposed synthesis technique provides the phase of the reflection coefficients which obtains the shaped beam required for this application, sectored in azimuth and squared cosecant in elevation. The accuracy and time efficiency of this technique are demonstrated using some results over a $30 \times 30$ element reflectarray illuminated by a feed horn. The radiation patterns of the entire antenna are shown and compared with the required LMDS templates.

This paper is organized as follows. Section 2 explains the scheme and characteristics of reflectarray antennas. In Section 3, the phase synthesis method is described, after being adapted to the reflectarray synthesis problem. Finally, Section 4 exposes the results for an LMDS base station application, and some conclusions are remarked in Section 5.

\section{Antenna Definition}

A planar reflectarray is used to illustrate the proposed technique. The scheme of the considered antenna configuration is shown in Figure 1. The reflectarray under study is a multilayer flat structure of printed stacked patches over a ground plane, with a horn antenna as primary feed. In order to account for both structures, two coordinate reference systems are established: a reflectarray reference system, whose origin is placed at the centre of the reflectarray surface and defined by $\left(\hat{x}_{R}, \hat{y}_{R}, \hat{z}_{R}\right)$, and a feed reference system located at the horn phase-centre and denoted by $\left(\hat{x}_{F}, \hat{y}_{F}, \hat{z}_{F}\right)$. The $\hat{z}_{F}$-axis identifies the pointing direction of the feed horn. Moreover, each reflectarray cell can be identified by the indexes $n=1 \cdots N$ and $m=1 \cdots M$, to describe its position in the $\hat{x}_{R}$-axis and the $\hat{y}_{R}$-axis, respectively, being $N, M$ the number of elements in each direction.

The feed horn illuminates the reflectarray and produces the incident field $E_{\text {inc }}^{X / Y}\left(x_{R}[n], y_{R}[m]\right)$ on each reflectarray cell (denoted for simplicity by $\left.E_{\text {inc }}^{X / Y}(n, m)\right)$. Although a nearfield can be used to accurately model the field radiated by the feed horn, a far-field simple model based on $\cos ^{q}(\theta)$ function of the feed pattern can be used [21]. Since the feed is

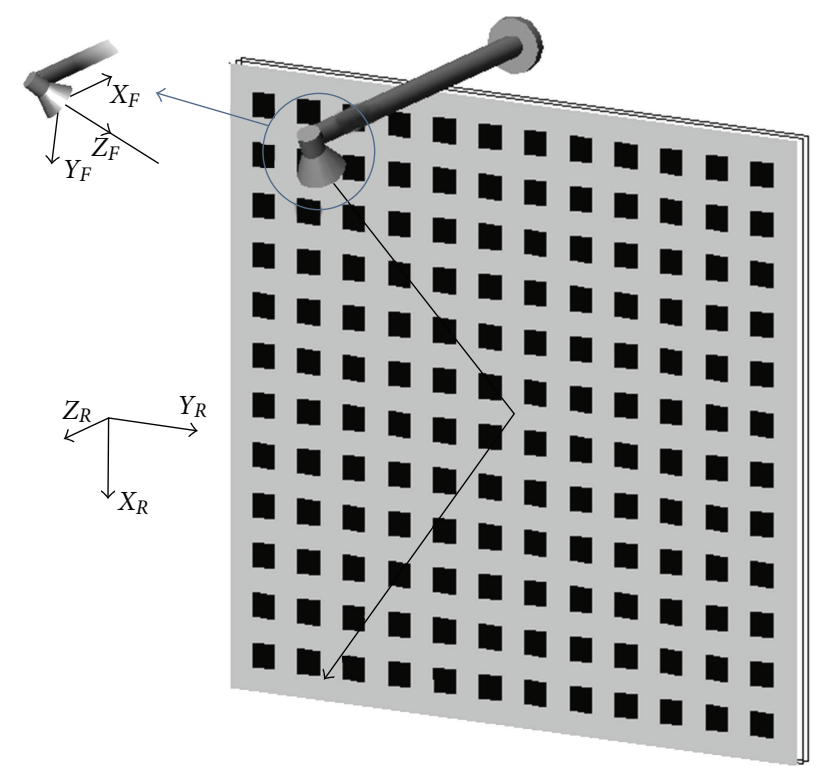

Figure 1: Scheme of the flat reflectarray.

TABle 1: Font sizes for papers values of $q$ factor used to model the feed horn.

\begin{tabular}{lccc}
\hline Frequency $(\mathrm{GHz})$ & 24.5 & 25.5 & 26.5 \\
\hline$q$-factor in E-plane & 33 & 35 & 37 \\
$q$-factor in H-plane & 37 & 39 & 41 \\
$q$ in $\cos ^{q}(\theta)$ (averaged) & 35 & 37 & 39 \\
\hline
\end{tabular}

a pyramidal horn, the beamwidth in E-plane and $\mathrm{H}$-plane is not the same, and therefore different $q$ values are estimated in each plane and an averaged value is used in the model. The $q$ factor also depends on the frequency and has been calculated at the central and extreme frequencies of the application band [7], see Table 1. A vertical and horizontal polarizations, $X_{R}$ and $Y_{R}$, can be considered, so the superindex $X / Y$ denotes this polarization of the feed, used to produce an electric field mainly in the $X_{R}$ or $Y_{R}$ direction. Note in Figure 1 that the vertical polarization corresponds to $X_{R}$-polarization, because the electric field in the $X_{R}$-direction is vertical, according to the considered reference system. For each polarization, the two components of the tangential electric field on the reflectarray surface are calculated, so the incident field is given by

$$
\vec{E}_{\text {inc }}^{X / Y}(n, m)=E_{x, \text { inc }}^{X / Y} \hat{x}_{R}+E_{y, \text { inc }}^{X / Y} \hat{y}_{R} .
$$

The field reflected on the reflectarray surface is used for the computation of the far-field radiation patterns of the entire antenna. The reflected field can be written as

$$
\vec{E}_{\text {ref }}^{X / Y}(n, m)=E_{x, \text { ref }}^{X / Y} \hat{x}_{R}+E_{y, \text { ref }}^{X / Y} \hat{y}_{R} .
$$

The relation between the incident and the reflected fields in each element $(n, m)$ of the reflectarray can be represented as

$$
\left[E_{x, \mathrm{ref}}^{X / \mathrm{Y}} \hat{x}_{R}, E_{y, \text { ref }}^{X / Y} \hat{y}_{R}\right]^{T}=\mathbf{R}(n, m) \cdot\left[E_{x, \text { inc }}^{X / Y} \hat{x}_{R}, E_{y, \text { inc }}^{X / Y} \hat{y}_{R}\right]^{T},
$$


where $(\cdot)^{T}$ indicates the transpose and $\mathbf{R}(n, m)$ is a complex matrix, which refers to the reflection coefficient of each reflectarray element:

$$
\mathbf{R}(n, m)=\left(\begin{array}{cc}
\rho_{x x}(n, m) & \rho_{x y}(n, m) \\
\rho_{y x}(n, m) & \rho_{y y}(n, m)
\end{array}\right)
$$

The $\mathbf{R}$ matrix characterizes the behavior of each reflectarray element. The components $\rho_{x x}(n, m)$ and $\rho_{y x}(n, m)$ are the direct and cross-reflection coefficients for an incident wave with the tangential component of the electric field in the $X_{R}$ direction, and $\rho_{y y}(n, m)$ and $\rho_{x y}(n, m)$ are the reflection coefficients for the $Y_{R}$ component. All of them depend on the angle of incidence of the impinging wave coming from the feed horn, and on the element geometry, adjusted to produce the required phase-shift, so that the matrix is different for each element of the reflectarray.

The calculation of the $\mathbf{R}$ matrix and the incident field given in (1) should be properly obtained. Considering the development in [9], the direct coefficient $\rho_{x x}(n, m)$ in (4) is the only used to calculate the copolar component of the radiation pattern when linear $X_{R}$-polarization is considered, since the term of $\rho_{x y}(n, m)$ can be neglected because of its low contribution to the total radiated field. The same criteria would be done over $\rho_{y y}(n, m)$ and $\rho_{y x}(n, m)$ for $Y_{R^{-}}$ polarization. Given this assumption, the copolar reflected field in each cell can be considered as

$$
\vec{E}_{\mathrm{ref}}^{X / Y}(n, m) \cong \rho_{x x}(n, m) \cdot E_{x, \text { inc }}^{X / Y}(n, m) \hat{x}_{R} .
$$

The phase of each reflection coefficient $\rho_{x x}(n, m)$ is achieved by adjusting the dimensions of the printed elements, and it must be obtained using an optimization method, as the technique presented in the next section.

\section{Phase Synthesis Method}

3.1. Far Field Radiation Pattern of the Reflectarray. Once the reflectarray antenna is described, the aim of this section is to obtain the phase of the reflection coefficient of each reflectarray element in order to fulfill the required pattern for the beam.

Considering the total field reflected by the reflectarray calculated in (5), the radiation pattern of the antenna is computed. Assuming the second principle of equivalence, the radiated far field is given by

$$
\vec{E}^{X / Y}(u, v)=\sum_{m=1}^{M} \sum_{n=1}^{N} \vec{E}_{\mathrm{ref}}^{X / Y}(n, m) e^{j n(2 \pi / \lambda) d_{x} u} e^{j m(2 \pi / \lambda) d_{y} v}
$$

where $u=\sin (\theta) \cos (\phi), v=\sin (\theta) \sin (\phi)$ are the elevation and azimuth angles, respectively, $\vec{E}^{X / Y}(u, v)$ is the far field produced by the entire antenna in $(u, v)$ direction, $\lambda$ is the wavelength in vacuum, and $d_{x}, d_{y}$ are the distances between radiating elements in the $\vec{x}_{R}$ and $\vec{y}_{R}$ axes of the reflectarray.

Let $\vec{E}_{0}^{X / Y}(u, v)$ be the radiation pattern of an element cell located at the origin of the coordinate system (the centre of the reflectarray), (6) can be rewritten using the formulation of the array factor as

$$
\begin{aligned}
\vec{E}^{X / Y}(u, v)= & \vec{E}_{0}^{X / Y}(u, v) \\
& \times \sum_{m=1}^{M} \sum_{n=1}^{N}\left(\left|\omega_{n m}\right| e^{j \varphi\left(\omega_{n m}\right)}\right) e^{j n(2 \pi / \lambda) d_{x} u} e^{j m(2 \pi / \lambda) d_{y} v} \\
= & \vec{E}_{0}^{X / Y}(u, v) A_{F}(u, v),
\end{aligned}
$$

where $\omega(n, m)$ can be considered as the excitation at element $(n, m)$, which determines how the reflection on each cell modifies the element radiation pattern $\vec{E}_{0}^{X / Y}(u, v)$, and $\varphi\left(\omega_{n m}\right)$ specifies its phase. Since the far field produced by the reflectarray is provided by the reflected distribution originated in the fed horn and considering (5), the magnitude $\left|\omega_{n m}\right|$ will be determined by the illumination of the primary feed as explained in the previous section. On the other hand, $\varphi\left(\omega_{n m}\right)$ represents the phase of the reflection coefficient $\rho_{x x}(n, m)$. Thus, in order to obtain the required phase terms $\varphi\left(\omega_{n m}\right)$ that modify the shape of the final radiation pattern, the synthesis process is only dependent on $A_{F}(u, v)$.

3.2. Phase Synthesis Using Least Squares Optimization and Levenberg-Marquardt Algorithm. The goal in many practical synthesis problems is obtaining a radiated far-field distribution concentrated between a set of bounds specified using a mask. One of the techniques for achieving this objective is the use of optimization, defining a cost function which penalizes the no-allowed field levels $[12,13]$. This function is given by defining the minimum and maximum bounds for the field values at each direction, and if only a set of $T$ possible directions $r_{t}=(u, v)_{t}, t=1 \cdots T$, is considered, this cost function can be expressed as

$$
\begin{aligned}
F=\sum_{t=1}^{T} F_{t}=\sum_{t=1}^{T} C_{t} & {\left[\left(G_{M}^{2}\left(r_{t}\right)-\left|\vec{E}^{X / Y}\left(r_{t}\right)\right|^{2}\right)\right.} \\
& \times\left(G_{m}^{2}\left(r_{t}\right)-\left|\vec{E}^{X / Y}\left(r_{t}\right)\right|^{2}\right) \\
& +\left.\left|G_{M}^{2}\left(r_{t}\right)-\right| \vec{E}^{X / Y}\left(r_{t}\right)\right|^{2} \mid \\
& \left.\times\left.\left|G_{m}^{2}\left(r_{t}\right)-\right| \vec{E}^{X / Y}\left(r_{t}\right)\right|^{2} \mid\right],
\end{aligned}
$$

where $G_{M}\left(r_{t}\right), G_{m}\left(r_{t}\right)$ are the maximum and minimum values specified for the field radiated at the direction $r_{t}$, $\vec{E}^{X / Y}\left(r_{t}\right)$ is defined in (7) and $C_{t} \in \mathbb{R}^{+}$is introduced to emphasize the error in some directions, useful when the field values are very low. $G_{M}\left(r_{t}\right), G_{m}\left(r_{t}\right)$, and $\vec{E}^{X / Y}\left(r_{t}\right)$ may be normalized in order to facilitate the optimization. $F_{t}$ in (8) represents the error at $r_{t}$, and it is null in those directions where the radiated field is inbounds, showing a higher value when an error exists: 


$$
F_{t}= \begin{cases}0, & \left.\forall t\left|G_{m}^{2}\left(r_{t}\right) \leq\right| \vec{E}^{X / Y}\left(r_{t}\right)\right|^{2} \leq G_{M}^{2}\left(r_{t}\right) \\ 2 C_{t}\left(G_{M}^{2}\left(r_{t}\right)-\left|\vec{E}^{X / Y}\left(r_{t}\right)\right|^{2}\right)\left(G_{m}^{2}\left(r_{t}\right)-\left|\vec{E}^{X / Y}\left(r_{t}\right)\right|^{2}\right), & \text { otherwise. }\end{cases}
$$

Operating over (9), the error addends in $F$ can be expressed as a sum of differences, so $F_{t}$ is written as

$$
F_{t}= \begin{cases}0, & \left.\forall t\left|G_{m}^{2}\left(r_{t}\right) \leq\right| \vec{E}^{X / Y}\left(r_{t}\right)\right|^{2} \leq G_{M}^{2}\left(r_{t}\right) \\ y_{t}-f_{t}(\boldsymbol{\alpha}), & \text { otherwise }\end{cases}
$$

where $y_{t}$ identifies the objective value for the direction $t$, and $f_{t}(\boldsymbol{\alpha})$ is the function to fit by calculating the set of parameters $\boldsymbol{\alpha}$, so that $F$ becomes minimal:

$$
\begin{gathered}
f_{t}(\boldsymbol{\alpha})=2 C_{t}\left|\vec{E}^{X / Y}\left(r_{t}\right)\right|^{2}\left(G_{M}^{2}\left(r_{t}\right)+G_{m}^{2}\left(r_{t}\right)-\left|\vec{E}^{X / Y}\left(r_{t}\right)\right|^{2}\right) \\
y_{t}=2 C_{t}\left(G_{M}^{2}\left(r_{t}\right) G_{m}^{2}\left(r_{t}\right)\right) .
\end{gathered}
$$

The vector $\boldsymbol{\alpha}$ contains the variables to be synthesized, that is, the phase values $\varphi\left(\omega_{n m}\right), n=1 \cdots N, m=1 \cdots M$, and it is represented as

$$
\begin{aligned}
& \boldsymbol{\alpha}=\left[\alpha_{1} \cdots \alpha_{p} \cdots \alpha_{P}\right], \quad p=1 \cdots P, \\
& \alpha_{p}=\operatorname{tg}\left(\varphi\left(\omega_{n m}\right)\right)=\frac{\operatorname{Im}\left(\omega_{n m}\right)}{\operatorname{Re}\left(\omega_{n m}\right)},
\end{aligned}
$$

where $P=N \cdot M$ is the dimension of $\boldsymbol{\alpha}$, and $\operatorname{Re}\left(\omega_{n m}\right), \operatorname{Im}\left(\omega_{n m}\right)$ are the real and imaginary parts of $\omega_{n m}$. In spite of the fact that the unknowns of the above problem are the phases $\varphi\left(\omega_{n m}\right)$, the elements of $\boldsymbol{\alpha}$ in (12) are the tangent of each phase, in order to avoid problems related to the periodicity in the solutions.

This synthesis process requires the use of an optimization algorithm able to minimize $F$. In this paper, the wellknown Levenberg-Marquardt (LM) algorithm [14] has been selected for this purpose, as a tradeoff between accuracy and simplicity. LM is an iterative algorithm able to solve certain nonlinear cost functions. One of the minimization problems where LM achieves good results arises in least squares. In order to apply the LM algorithm to (8), the cost function must be expressed as a least squares quadratic function, so the real cost function to implement needs to be defined as

$$
F_{\mathrm{LM}}=\sum_{t=1}^{T} F_{t}^{2}
$$

where $F_{t}$ is defined in (10). Note that the meaning of $F$ and $F_{\mathrm{LM}}$ are the same, even though the squared addends.

The LM method requires the calculation of a Jacobian matrix, denoted as $\mathbf{J}$, so that $F_{\mathrm{LM}}$ can be minimized. It is a $T \times P$ matrix whose elements are calculated using the partial derivatives over $F_{t}$, respect to each element of $\boldsymbol{\alpha}$. Thus, considering (10), each element of $\mathbf{J}$ is either null (for those $t$ th rows where $\left|\vec{E}^{X / Y}\left(r_{t}\right)\right|$ is in bounds, i.e., $F_{t}=0$ ) or defined as

$$
\begin{aligned}
\mathbf{J}(t, p)=\left.\frac{\partial \mathbf{f}}{\partial \boldsymbol{\alpha}}\right|_{t, p}=\frac{\partial f_{t}(\boldsymbol{\alpha})}{\partial \alpha_{p}} & \\
=4 C_{t}\left|\vec{E}_{0}^{X / Y}\left(r_{t}\right)\right|^{2}[ & \operatorname{Re}\left(A_{F}\left(r_{t}\right)\right) \frac{\partial \operatorname{Re}\left[A_{F}\left(r_{t}\right)\right]}{\partial \alpha_{p}} \\
& \left.+\operatorname{Im}\left(A_{F}\left(r_{t}\right)\right) \frac{\partial \operatorname{Im}\left[A_{F}\left(r_{t}\right)\right]}{\partial \alpha_{p}}\right] \\
& \quad\left[\left(G_{M}^{2}\left(r_{t}\right)+G_{m}^{2}\left(r_{t}\right)\right)-2\left|\vec{E}^{X / Y}\left(r_{t}\right)\right|^{2}\right]
\end{aligned}
$$

in those directions $r_{t}$ where $\left|\vec{E}^{X / Y}\left(r_{t}\right)\right|^{2}<G_{m}^{2}\left(r_{t}\right)$ or $\left|\vec{E}^{X / Y}\left(r_{t}\right)\right|^{2}>G_{M}^{2}\left(r_{t}\right)$. Note that $\left|A_{F}\left(r_{t}\right)\right|^{2}=\left[\operatorname{Re}\left(A_{F}\left(r_{t}\right)\right)\right]^{2}+$ $\left[\operatorname{Im}\left(A_{F}\left(r_{t}\right)\right)\right]^{2}$, and each partial derivate is directly demonstrated from (7) as

$$
\begin{aligned}
\frac{\partial \operatorname{Re}\left(A_{F}\left(r_{t}\right)\right)}{\partial \alpha_{p}}= & \sum_{m=1}^{M} \sum_{n=1}^{N}-\operatorname{Re}\left(\omega_{n m}\right) \\
\times & {\left[\cos \left(n \frac{2 \pi}{\lambda} d_{x} u\left(r_{t}\right)\right) \sin \left(m \frac{2 \pi}{\lambda} d_{y} v\left(r_{t}\right)\right)\right.} \\
& \left.+\sin \left(n \frac{2 \pi}{\lambda} d_{x} u\left(r_{t}\right)\right) \cos \left(m \frac{2 \pi}{\lambda} d_{y} v\left(r_{t}\right)\right)\right], \\
\frac{\partial \operatorname{Im}\left(A_{F}\left(r_{t}\right)\right)}{\partial \alpha_{p}}= & \sum_{m=1}^{M} \sum_{n=1}^{N} \operatorname{Re}\left(\omega_{n m}\right) \\
\times & {\left[\cos \left(n \frac{2 \pi}{\lambda} d_{x} u\left(r_{t}\right)\right) \cos \left(m \frac{2 \pi}{\lambda} d_{y} v\left(r_{t}\right)\right)\right.} \\
& \left.\quad-\sin \left(n \frac{2 \pi}{\lambda} d_{x} u\left(r_{t}\right)\right) \sin \left(m \frac{2 \pi}{\lambda} d_{y} v\left(r_{t}\right)\right)\right],
\end{aligned}
$$

where $u\left(r_{t}\right), v\left(r_{t}\right)$ represent the $u, v$ values determined by the $r_{t}$ direction. Once the Jacobian matrix is calculated, the LM algorithm can be applied iteratively over $F_{\mathrm{LM}}$ as

$$
\left[\mathbf{J}_{i}^{T} \cdot \mathbf{J}_{i}+\mu_{i} \cdot \operatorname{diag}\left(\mathbf{J}_{i}^{T} \cdot \mathbf{J}_{i}\right)\right] \cdot \boldsymbol{\delta}_{i}=\mathbf{J}_{i}^{T} \cdot \mathbf{F}_{t, i}
$$

where the subindex $i$ represents each iteration, $\operatorname{diag}(\cdot)$ is the diagonal matrix, $\mathbf{J}_{i}$ is the Jacobian matrix of $\boldsymbol{\alpha}_{i}$ (in each iteration $i), \mu_{i}$ is a convergence parameter that depends on $i$, and $\mathbf{F}_{t, i}$ is a $T \times 1$ vector which represents the $T$ samples of $F_{t}$ in (9) for each $i$. Note that either $\mathbf{J}_{i}$ or $\mathbf{F}_{t, i}$ present null samples which do not contribute in the optimization, 


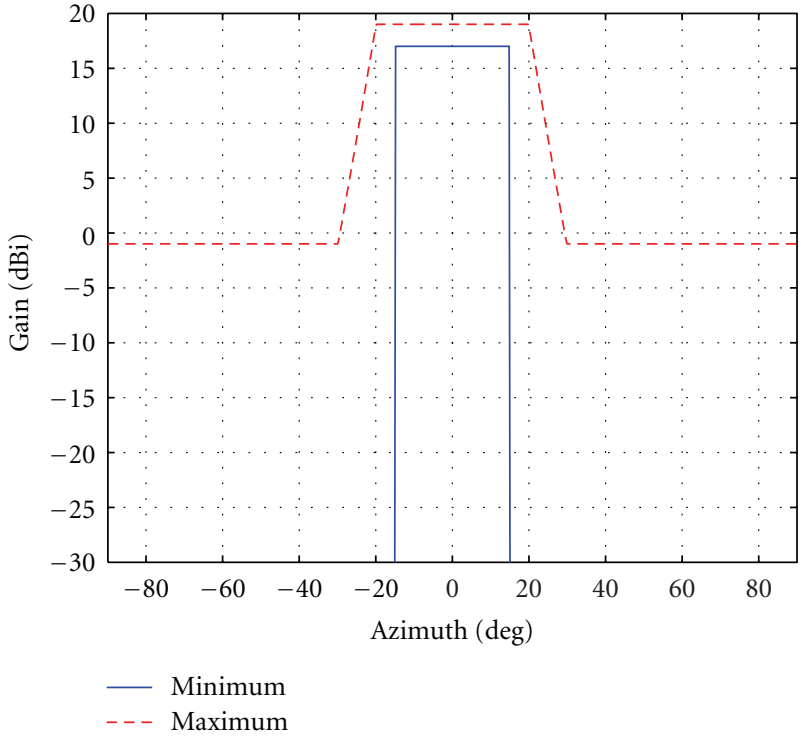

(a)

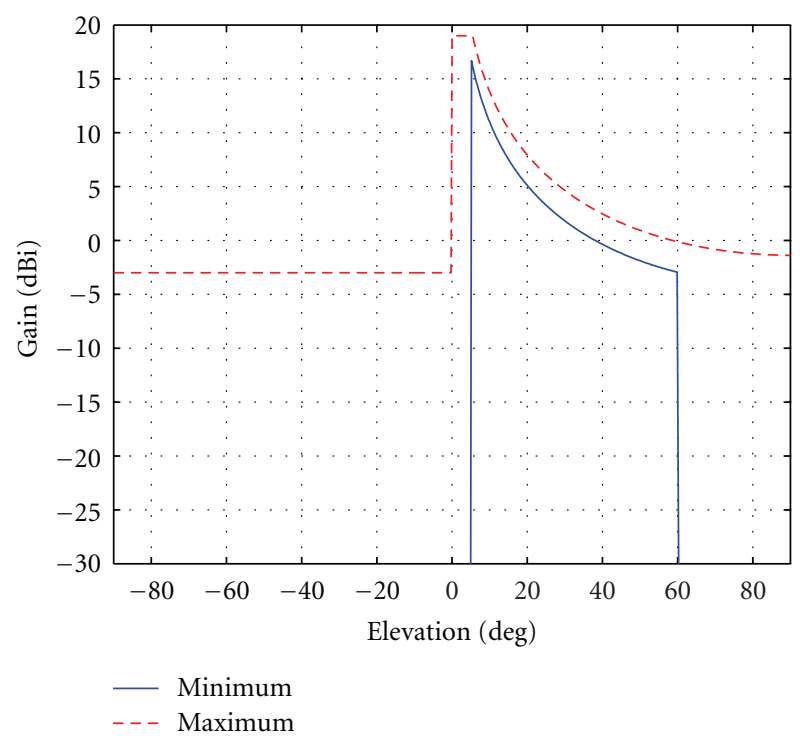

(b)

FIgURE 2: Pattern requirements in azimuth (a) and elevation (b). Maximum (dashed lines) and minimum (solid lines) templates.

because of the fact that there is no error in those directions; moreover, the directions $r_{t}$ where the field is inbounds are different depending on the iteration, and should increase as the algorithm progresses. Finally, $\boldsymbol{\delta}_{i}$ is an update vector satisfying the equality, which is calculated as the solution of the normal equation associated to the previous least square problem as

$$
\boldsymbol{\delta}_{i}=\left(\mathbf{B}_{i}^{T} \cdot \mathbf{B}_{i}\right)^{-1} \cdot \mathbf{B}_{i}^{T} \cdot \mathbf{b}_{i}
$$

where matrix $\mathbf{B}_{i}=\left[\mathbf{J}_{i}^{T} \cdot \mathbf{J}_{i}+\mu_{i} \cdot \operatorname{diag}\left(\mathbf{J}_{i}^{T} \cdot \mathbf{J}_{i}\right)\right]$, vector $\mathbf{b}_{i}=$ $\mathbf{J}_{i}^{T} \cdot \mathbf{F}_{t, i}$, and $(\cdot)^{-1}$ indicates the inverse matrix. Vector $\boldsymbol{\delta}_{i}$ is iteratively updating the solution as

$$
\boldsymbol{\alpha}_{i+1}=\boldsymbol{\alpha}_{i}+\boldsymbol{\delta}_{i} .
$$

Note that the solution for the phases in each iteration is given by $\operatorname{tg}^{-1}\left(\boldsymbol{\alpha}_{i+1}\right)$ according to (12).

LM requires certain operations over a high dimensional matrix $\mathbf{J}_{i}$. The Jacobian matrix inversion may have an important temporal cost, or an ill-conditioned matrix $\mathbf{J}_{i}$ might lead to instability in (17). As a result, a gradient descent technique, such as the Conjugate Gradient Squared (CGS) method [22], is implemented to calculate $\boldsymbol{\delta}_{i}$ (given $\mathbf{B}_{i}$ and $\mathbf{b}_{i}$ ) and prevent this issue.

In order to achieve convergence, the starting parameters should be chosen to provide a valid solution. As it will be shown in the next section, the priori knowledge of the LMDS bounds can provide initial values of $\boldsymbol{\alpha}$ which accelerates the optimization process. Moreover, the parameter $\mu$ controls the speed of convergence, so it must be selected carefully to avoid divergence. It defines the steps size to achieve the minimum, closer to the gradient descent direction. One of the most extended techniques to choose this parameter consists in starting with $\mu_{0}$ proportional to the maximum value of $\operatorname{diag}\left(\mathbf{J}_{i}^{T} \cdot \mathbf{J}_{i}\right)$ [18], and defining a real parameter $\beta>1$, so that $\mu_{i+1}=\mu_{i} \beta$ or $\mu_{i+1}=\mu_{i} / \beta$ if the cost function increases or decreases, respectively, each iteration $i$ [19]. The high number of variables in the current reflectarray problem requires an exhaustive control of $\mu_{i}$, so its increase is only allowed when the cost function decreases in more than five iterations consecutively, forcing a decrease every time $F_{\mathrm{LM}, i}$ goes higher. Note that $\mu_{i}=0$ converts LM into the Gauss-Newton's method [19], easy to diverge in a nonlinear problem with a high number of unknowns, so this value must be disregarded.

\section{Simulations and Results}

In this section, the proposed technique is demonstrated by the phase-only synthesis of a reflectarray designed for LMDS applications. This antenna must be synthesized to fulfill the coverage specifications of a central station of LMDS service in the $24.5-26.5 \mathrm{GHz}$ band, where a shaped beam both in elevation (squared cosecant) and in azimuth (sectored) [11] is required. The templates of minimum and maximum requirements are shown in Figures 2 and 3. To achieve this beam shaping and gain, a reflector surface is defined. Since the bandwidth of the application is about $8 \%(24.5$ to $26.5 \mathrm{GHz}$ ), a two-layer printed reflectarray working at central frequency of the band $(25.5 \mathrm{GHz})$ is considered [9]. The grating lobes are avoided or negligible in the entire frequency band. This flat reflectarray is composed of $30 \times 30$ rectangular patches of variable size arranged in a regular mesh, with a periodic cell $d_{x} \times d_{y}$ defined as $5.88 \times 5.88 \mathrm{~mm}$ (half a wavelength at the central frequency). The phase centre of the feed-horn is placed at $(-94,0,214) \mathrm{mm}$ in the reflectarray reference system. The resulting $f / D$ of the antenna is 1.2. 


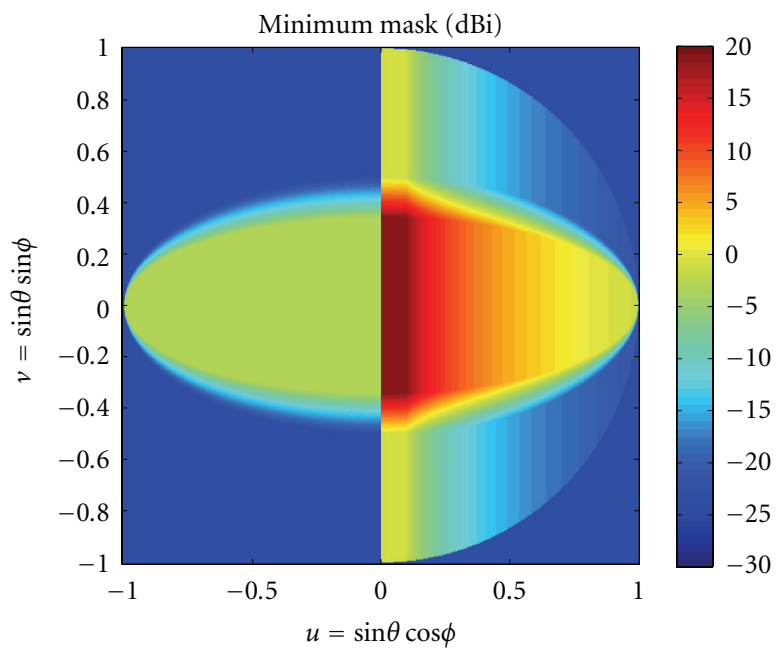

(a)

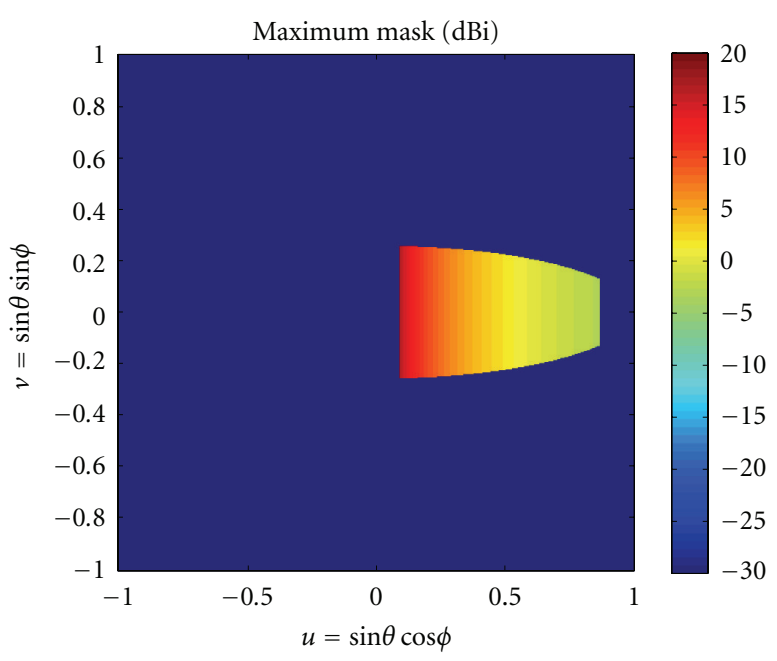

(b)

Figure 3: Pattern requirements $G_{M}\left(r_{t}\right)$ (a) and $G_{m}\left(r_{t}\right)(\mathrm{b})$, in all the considered directions $r_{t}=(u, v)_{t}$.

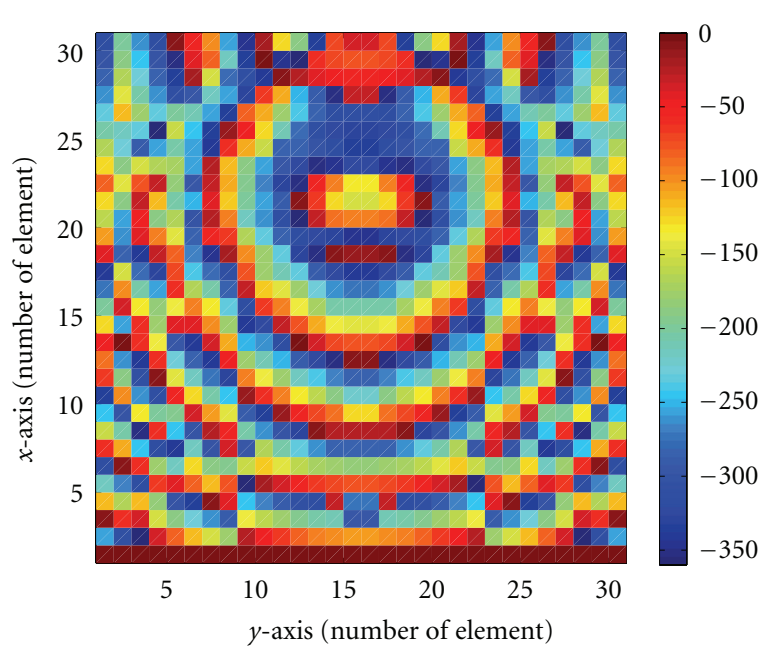

FIGURE 4: Synthesized phase distribution of the reflection coefficient for the $X_{R}$-polarization (degrees).

The phase distribution of the reflection coefficient is obtained using the method described in Section 3. The incident field that defines $\left|\omega_{n m}\right|, n=1 \cdots N, m=1 \cdots M$, is determined by the $\cos ^{q}(\theta)$ (see Table 1), and the bounds $G_{M}\left(r_{t}\right), G_{m}\left(r_{t}\right)$ are the required in LMDS [11], shown in Figure 3.

The resulting phase distribution for $X_{R}$-polarization after applying the technique that is discussed in the previous section is shown in Figure 4, being the corresponding to $Y_{R^{-}}$ polarization very similar. The small difference between both phase distributions is the result of slight differences in the incident field for both linear polarizations.

This solution has been obtained after 3900 iterations of the proposed algorithm, where $T=16384$ considered directions (taking 128 samples uniformly in $u, v \in[-1,1]$ ), and less than a minute per iteration on an Intel Core 2
Duo PC with $2.4 \mathrm{GHz}$ processor. The parameter $C_{t}=$ $1 /\left(G_{M}\left(r_{t}\right)-G_{m}\left(r_{t}\right)\right)$, so the directions with demanding restrictions are more penalized. The convergence parameter has been empirically set at $\mu_{0}=5$, a value which allows a soft descent toward the solution. This value has been modified as it was explained in 3.1 , with $\beta=1.2$, so $\mu \epsilon(0.13,331.24)$ in the different iterations of the algorithm. On the other hand, the starting point of the synthesis process (initial phase distribution) is chosen to have a high value at the maximum of the azimuth and elevation masks, which allows a useful initial point to begin the optimization process. The algorithm stops at iteration 3900, when $F_{\mathrm{LM}, i}<F_{\mathrm{LM}, i-50} / 2$ and $F_{\mathrm{LM}, i}<$ 0.01 . The final error in the synthesis process is $F_{\mathrm{LM}}=0.56$. $10^{-2}$, and $\overline{F_{\mathrm{LM}}}=F_{\mathrm{LM}} / T=3.42 \cdot 10^{-7}$ if all directions $T$ are considered, even the ones with no error, which means that the field is inbounds in most of the directions. It is a low value, which represents the convergence of the iterative algorithm.

The obtained phase distribution is used to calculate the three-dimensional radiation pattern, which has been simulated at the central frequency of the band $(25.5 \mathrm{GHz})$, for dual linear polarization (vertical and horizontal polarizations), see Figure 5. The final phases for each polarization $X / Y$ are not equal, due to the difference in the illumination of the reflectarray produced by the feed for the two polarizations. Note that the projection of the incident field on the reflectarray cells is different for the two orthogonal linear polarizations.

Main cuts of the beams (elevation and azimuth) are also depicted in Figure 6. Although ideal phase-shift elements have been assumed as reflectarray elements, the radiation patterns have been computed considering the illumination and spillover efficiency of the antenna and they are given in gain $(\mathrm{dBi})$. The results show good agreement with requirements in both polarizations, particularly in the coverage 


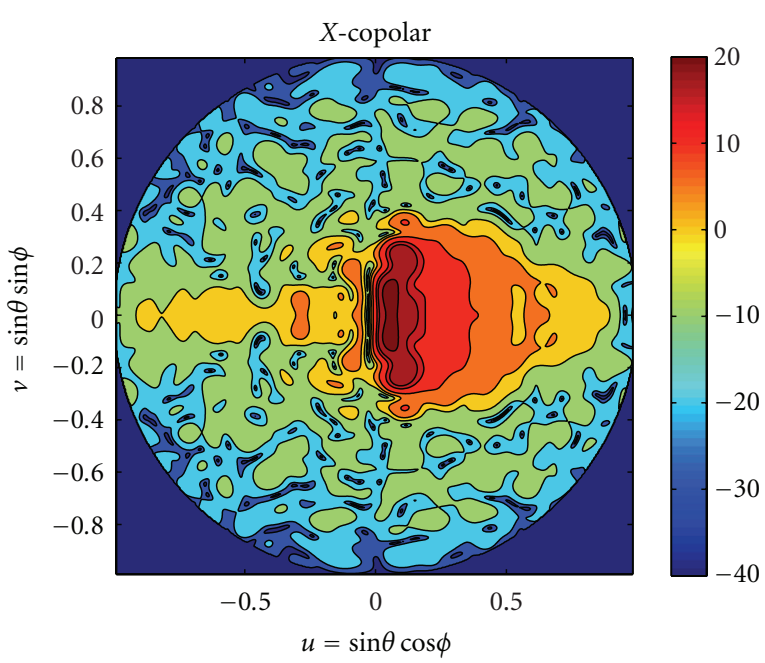

(a)

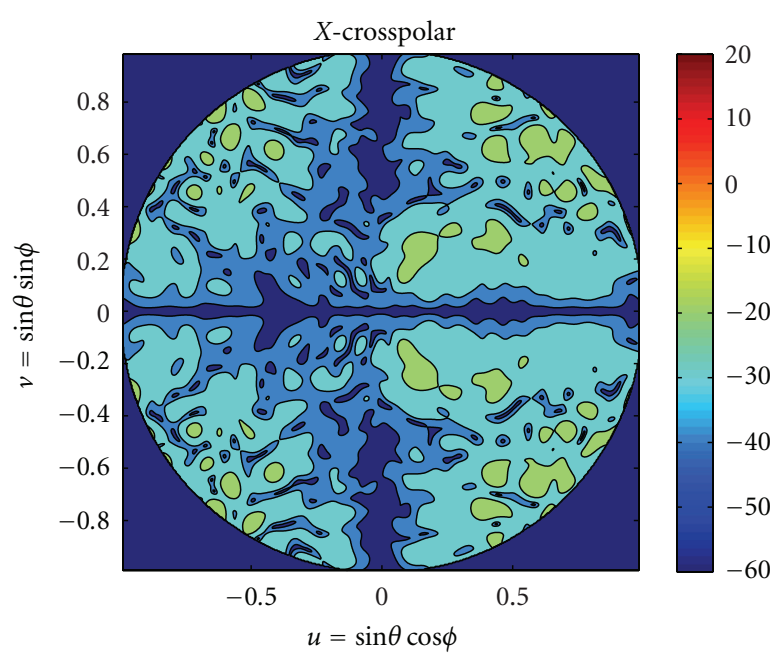

(c)

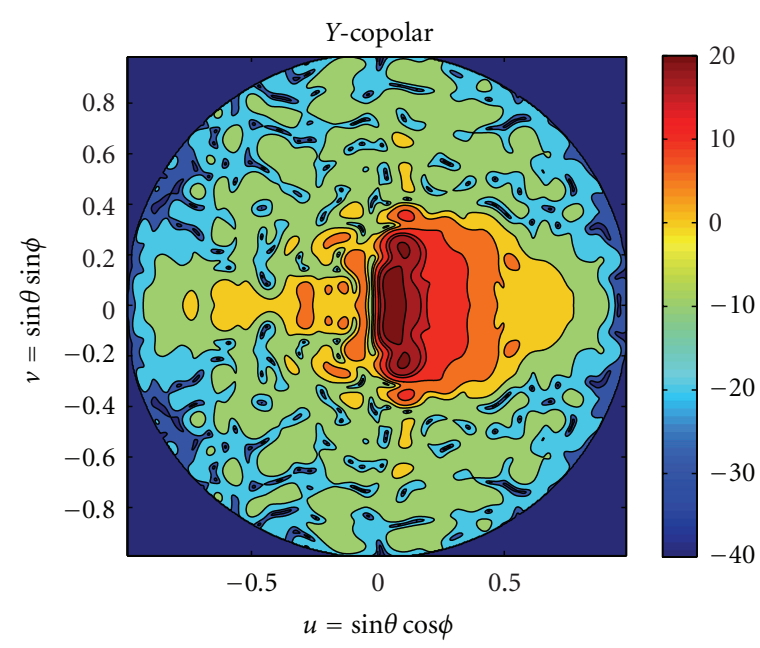

(b)

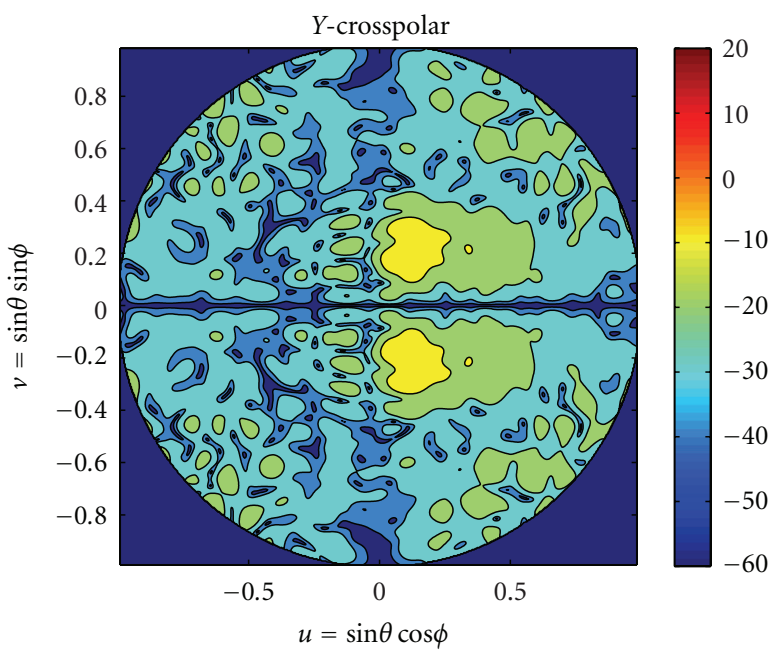

(d)

Figure 5: Simulated three-dimensional radiation patterns considering the synthesized phase distribution; vertical (a and c) and horizontal (b and d) polarizations for copular ( $\mathrm{a}$ and $\mathrm{b}$ ) and crosspolar ( $\mathrm{c}$ and $\mathrm{d}$ ) components.

regions. The beam shaping is achieved, and the gain requirements are fulfilled in a high percentage of the coverage. However, small disagreement is found in noncoverage zone but with low side-lobe level. In elevation (Figure 6(b)), the beam shaping requirement is more restrictive and shows a higher gain than required or some ripple is obtained in the region defined by negative elevation angles. Moreover, those negative elevation angles are outside the coverage region of the central station and some error can be acceptable. The pointing directions towards the Earth surface are defined by the positive elevation angles, which correspond to the squared cosecant pattern. Thus, this error is not critical for the station performances. The most critical direction is the zero angle in elevation, where an interference with other LMDS base stations may exist. However, the radiation pattern in this direction produces a deep null, avoiding the potential interference. In spite of those limitations, the squared cosecant in elevation is properly achieved and the radiated field is inbounds in almost all the pointing angles. Moreover, the obtained results are similar in quality to LMDS radiated patterns achieved with traditional methods [9], with the advantage of the use of a systematic framework for optimizing the required phases. These features demonstrate the success of the proposed method.

\section{Conclusions}

A phase-only synthesis technique for shaped beams has been discussed and proposed for the systematic synthesis of reflectarrays, providing a framework suitable for general reflectarrays synthesis problems. It has been shown to lead to excellent results when used in the presented examples with antennas in LMDS central stations or Direct Broadcast Satellite (DBS) antennas applications.

Using a cost function properly defined and the Levenberg-Marquardt optimization algorithm, the reflection 


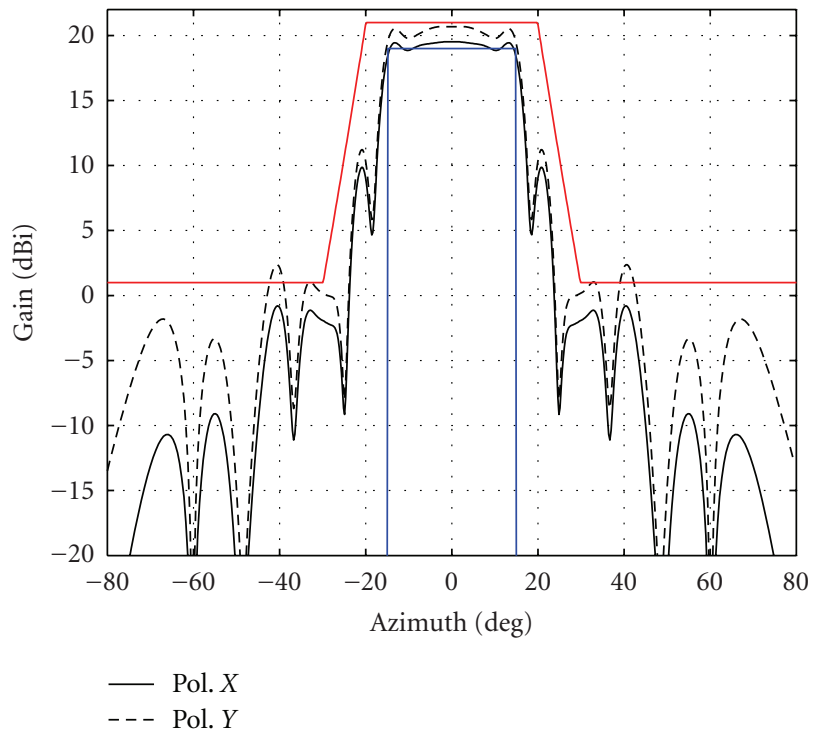

(a)

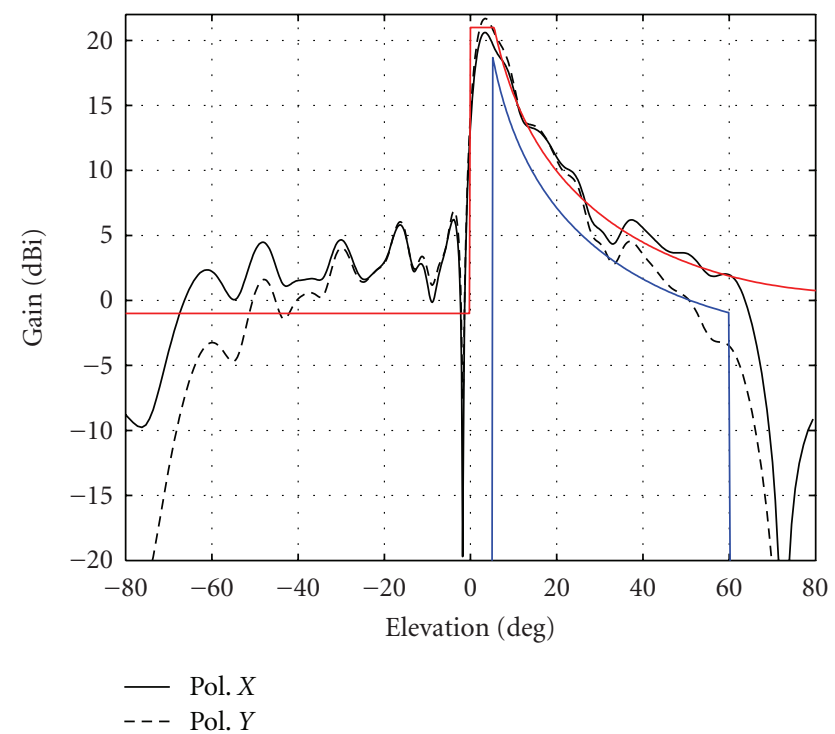

(b)

FIGURE 6: Simulations (vertical and horizontal polarizations) at the central frequency. Main cuts in azimuth (a) and elevation (b), compared with the maximum and minimum required gains (solid red and blue lines).

coefficients of each reflectarray element are obtained. The technique has been applied to the synthesis of a LMDS central station antenna covering 30-degree azimuth sector. The results match with the application requirements. Moreover, the required squared cosecant in the elevation plane is properly obtained, which demonstrates the capabilities of the proposed method to synthesize stringent-shaped beams with low time requirements. Thus, avoiding either the combination of other synthesis techniques or the control on the algorithm while the optimization progresses, the proposed framework provides proper results in LMDS applications.

Finally, this phase-only synthesis technique can be applied to other reflectarray configurations, with more feed horns or different number of reflectarray elements. Only defining the incident beam and specifying the needed bounds, this method provides the synthesized values which fulfill demanding requirements without the need of any adaptation of the method.

\section{Acknowledgments}

This work has been supported by the Ministerio de Ciencia e Innovación of Spain/FEDER under Projects CONSOLIDERINGENIO CSD2008-00068 and TEC2011-24492; by the Gobierno del Principado de Asturias (PCTI)/FEDER-FSE under Projects EQUIP08-06, FC09-COF09-12, EQUIP1031, and PC10-06 (Flexant); Grant BP10-165; by Cátedra Telefónica-Universidad de Oviedo.

\section{References}

[1] J. Huang and J. A. Encinar, Reflectarray Antennas, IEEE Press/Wiley, Piscataway Township, NJ, USA, 2007.
[2] A. Jacomb-Hood and E. Lier, "Multibeam active phased arrays for communicatons satellites," IEEE Microwave Magazine, vol. 1, no. 4, pp. 40-47, 2000.

[3] L. Schulwitz and A. Mortazawi, "A compact dual-polarized multibeam phased-array architecture for millimeter-wave radar," IEEE Transactions on Microwave Theory and Techniques, vol. 53, no. 11, pp. 3588-3594, 2005.

[4] P. Balling, K. Tjonneland, L. Yi, and A. Lindley, "Multiple contoured beam reflector antenna systems," in Proceedings IEEE Antennas and Propagation Society International Symposium, Ann Arbor, Mich, USA, June 1993.

[5] W. Menzel, M. Al-Tikriti, and R. Leberer, "A 76 GHz multiplebeam planar reflector antenna," in Proceeding of the 32nd European Microwave Conference, pp. 977-980, Milano, Italy, October 2002.

[6] J. A. Encinar, L. S. Datashvili, J. A. Zornoza et al., "Dualpolarization dual-coverage reflectarray for space applications," IEEE Transactions on Antennas and Propagation, vol. 54, no. 10, pp. 2827-2837, 2006.

[7] J. A. Encinar, M. Arrebola, L. F. de la Fuenta, and G. Toso, "A transmit-receive reflectarray antenna for direct broadcast satellite applications," IEEE Transactions on Antennas and Propagation, vol. 59, no. 9, pp. 3255-3264, 2011.

[8] A. Trastoy, J. A. Rodríguez, F. Ares, J. Vassal'lo, and E. Moreno, "University of santiago de compostela contribution to ARCO project: synthesis of footprint patterns for planar reflectarrays," in Proceedings of the 18th International Conference on Applied Electromagnetics and Communications (ICECom '05), October 2005.

[9] M. Arrebola, J. A. Encinar, and M. Barba, "Multifed printed reflectarray with three simultaneous shaped beams for LMDS central station antenna," IEEE Transactions on Antennas and Propagation, vol. 56, no. 6, pp. 1518-1527, 2008.

[10] A. Chakraborty, B. N. Das, and G. S. Sanyal, "Beam shaping using nonlinear phase distribution in a uniformly spaced array," IEEE Transactions on Antennas and Propagation, vol. 30, no. 5, pp. 1031-1034, 1982. 
[11] ETSI EN 301215 v1.3.1, “European standard (Telecommunications series), fixed radio systems, pointto-multipoint antennas, antennas for point-to-multipoint fixed radio systems in the $11 \mathrm{GHz}$ to $60 \mathrm{GHz}$ band, Part $2: 24 \mathrm{GHz}$ to $30 \mathrm{GHz}$, Standard," 2002.

[12] F. Las-Heras and T. K. Sarkar, "A direct optimization approach for source reconstruction and NF-FF transformation using amplitude-only data," IEEE Transactions on Antennas and Propagation, vol. 50, no. 4, pp. 500-510, 2002.

[13] F. Las-Heras, B. Galocha, and J. L. Besada, "Equivalent source modelling and reconstruction for antenna measurement and synthesis," in Proceedings of the IEEE Antennas and Propagation Society International Symposium, pp. 156-158, Montreal, Canada, July 1997.

[14] J. Nocedal and S. J. Wright, Numerical Optimization, Springer, 2nd edition, 2006.

[15] S. C. Chapra and R. P. Canale, Numerical Methods for Engineers, McGraw Hill, 1985.

[16] D. E. Goldberg, Genetic Algorithms for Search, Optimization, and Machine Learning, Addison-Wesley Longman Publishing, Reading, Mass, USA, 1989.

[17] K. V. Price, R. M. Storn, and J. A. Lampinen, Differential Evolution: A Practical Approach to Global Optimization, Springer, Berlin, Germany, 2006.

[18] K. Madsen, H. B. Nielsen, and J. Sondergaard, "Robust subroutines for non-linear optimization," Tech. Rep. IMMREP-2002-02, Technical University of Denmark, 2002.

[19] T. H. Ismail, D. I. Abu-Al-Nadi, and M. J. Mismar, "Phaseonly control for antenna pattern synthesis of linear arrays using the Levenberg-Marquardt algorithm," Electromagnetics, vol. 24, no. 7, pp. 555-564, 2004.

[20] Y. Wen, W. S. Gan, and J. Yang, "Nonlinear least-square solution to flat-top pattern synthesis using arbitrary linear array," Signal Processing, vol. 85, no. 9, pp. 1869-1874, 2005.

[21] M. Arrebola, Y. Álvarez, J. A. Encinar, and F. Las-Heras, "Accurate analysis of printed reflectarrays considering the near field of the primary feed," IET Microwaves, Antennas and Propagation, vol. 3, no. 2, pp. 187-194, 2009.

[22] A. V. Knyazev and I. Lashuk, "Steepest descent and conjugate gradient methods with variable preconditioning," Journal on Matrix Analysis and Applications, vol. 29, no. 4, pp. 1267-1280, 2007. 

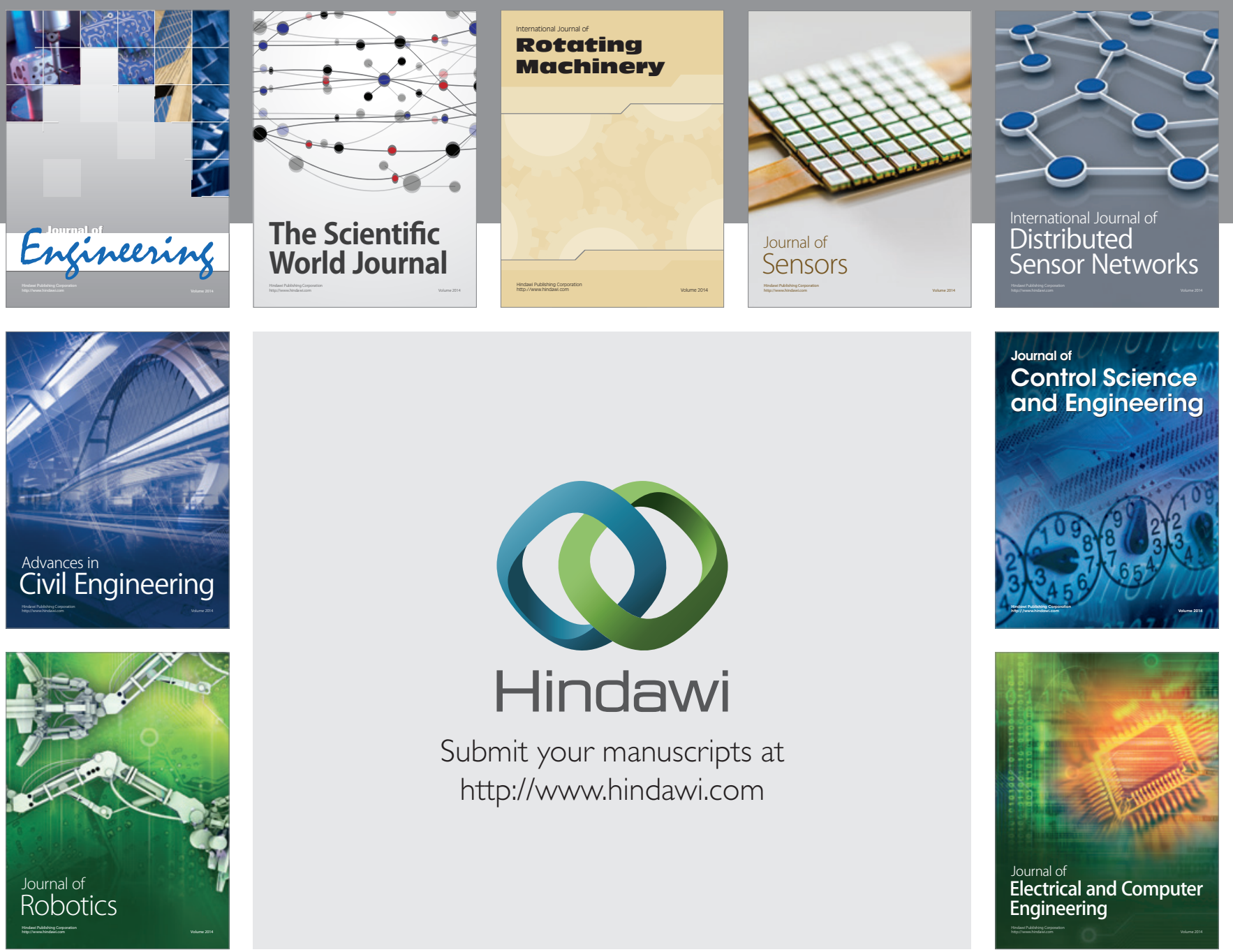

Submit your manuscripts at

http://www.hindawi.com
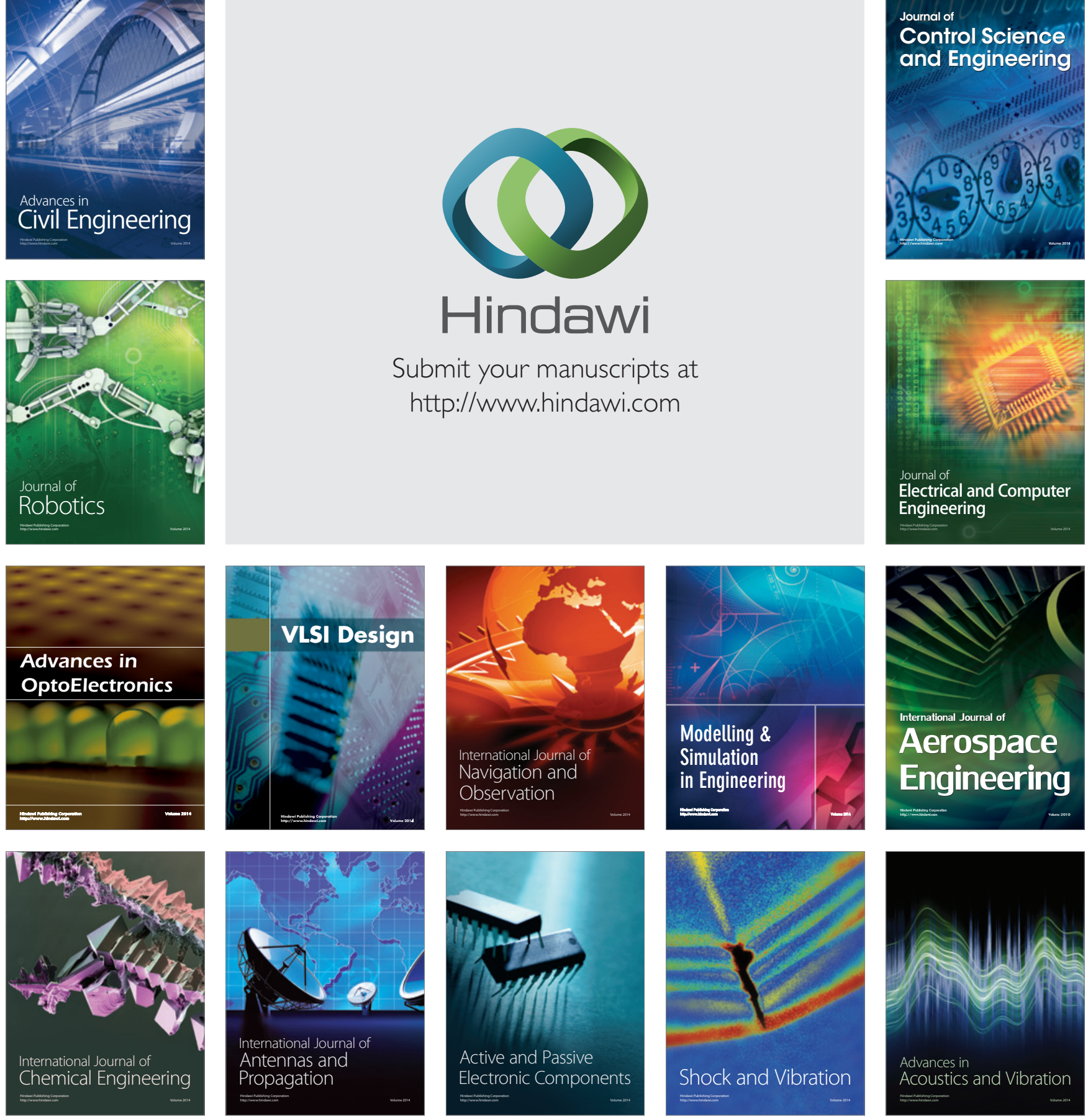